\title{
Maximizing Your Success with Telehealth
}

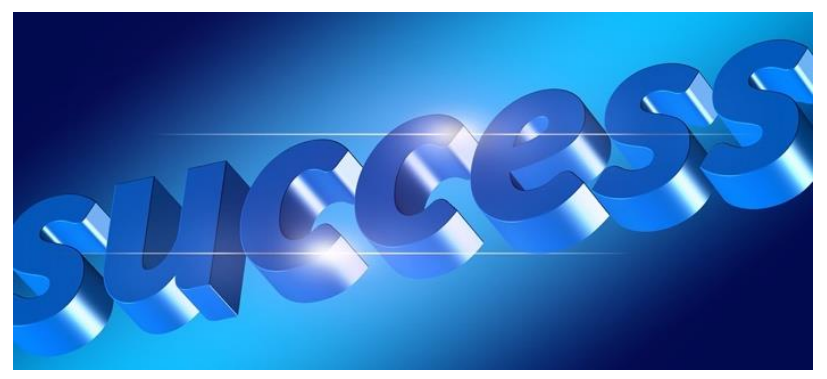

Jeffrey Jones

Many health systems are making significant investments in telehealth with grand goals of improving care delivery, increasing care capacity and reducing delivery costs. However, some organizations are not positioned well to achieve these goals. The Project Management Institute (PMI) states that $44 \%$ of strategic initiatives will fail, and will do so for a multitude of reasons. ${ }^{1}$ Telehealth is a strategic imperative for most health delivery organizations (HDO), yet the chances of missing the targeted goals exist given the multiple stakeholders, numerous service options available and other complexities associated with telehealth.

We have worked with many health systems to identify, plan, design, deploy and operate telehealth programs and individual virtual-enabled clinical services. We have witnessed successful telehealth programs as well as less than successful programs, and advised HDO's on improving telehealth results and outcomes. We find it is not WHAT HDOs are doing with telehealth that limits their value and success but HOW they are doing it.

There are many common threads that characterize a successful telehealth program (Table 1.). What follows is a discussion of success factors and suggestions on how to improve your program for greater performance. The ten success factors covered here include a clear strategy, executive and physician involvement, well defined and documented services, integration of services, focus on the patient and provider, accountability, focus on growth, clear success criteria, and integration with informatics technologies I may also reinforce what you already know to help ensure your success with telehealth. 
Table 1. The ten factors that characterize a successful telehealth program

\section{Factors}

1. Clear strategy

2. Executive and physician involvement

3. Services are defined and documented

4. Integration of services

5. Patient and provider focused

6. Accountability

7. Focus on growth

8. Clear success criteria

9. Oversight

10. Integration with informatics technologies

The telehealth program has a clear strategy, plan, and timeline aligned with strategic and operational imperatives of the health system

Each telehealth service should be unique to the health system, the individual needs, goals of the organization, and the patients served. There is no "one size fits all" telehealth service. Each organization's goals are different.

The telehealth services should reflect specific needs. A telehealth business plan should be created for each service and define realistic financial and non-financial benefits and expectations from the service. Many HDOs pursue telehealth initiatives based on nonfinancial, value-based opportunities and understand there is no quick return on investment.

Key executives and physicians are equally involved in development, growth, and success of the telehealth program

Executives and physicians must serve as champions to the organization, evangelize and stimulate support for and adoption of telehealth, and identify concerns and improvement opportunities. 
They understand there will be lessons learned from the initial phase and improve based on those lessons. Telehealth adoption starts in the planning stage of the service, and physician- and executive-leaders are vital to achieving adoption.

\section{Individual telehealth services are defined and documented}

The details of each service must be appropriately communicated to all who are affected, and they are aware of what is in scope and out-of-scope for the telehealth service.

Stakeholders have input into the design and operation of the service. Once defined, telehealth service education and training is provided to physicians and providers, which is targeted and relevant to what they need to know.

\section{The telehealth service is integrated into daily care and workflow}

Telehealth is used when it supports and complements patient care, and is not used when in-person, "hands on" care is required. Telehealth is not viewed as a "stand-alone" use case with a separate care team or organization providing the service; telehealth becomes part of daily care methods. Ultimately, telehealth will become known as health care.

\section{Each telehealth service is focused on patient and physician/provider needs, rather than on technology}

The technology is intuitive and easy-to-use, flexible, and adjust to differing patient needs. Telehealth must provide a positive patient experience.

There is a dedicated telehealth team that is accountable for and focused on the results, outcomes and success of telehealth

The telehealth team works closely with the clinical service lines, physicians, and leadership to ensure expectations are achieved and accountability for expectations are maintained. 
The telehealth team provides education, training and support to sustain and grow telehealth operations. And the team proactively manages telehealth risk and market changes for the health system and plans new telehealth services.

\section{Focus is on growing the volume of telehealth encounters}

The team is realistic and understands that volume is grown incrementally (when using an organic growth approach) and occurs when physicians, providers and patients are comfortable and satisfied with the service.

Clear and unambiguous success criteria and key performance indicators (KPIs) for the telehealth service that are measured and reported on a consistently KPIs cover clinical, operational, financial, and technical elements. Adjustments are made to the service, as needed, based on quantitative and qualitative data.

A clinical care quality oversight committee is created and performs case reviews and quality oversight ensuring that clinical care quality is not deleteriously impacted by telehealth

Whether care is provided in person or via telehealth, variation is limited and standardized protocols and procedures are used.

Telehealth is integrated with the organization's health informatics technologies To achieve high-quality patient care, clinical information must be leveraged across the care continuum. Silos of information limit quality care.

\section{Conclusion}

As healthcare continues to go through a tectonic shift in the delivery, consumption, and payment for care, telehealth will play a significant role to enable more convenient, efficient and qualitative methods for care. When done successfully, telehealth provides increased value to patients and providers. Weaving these 10 threads into your telehealth program will help ensure success. 


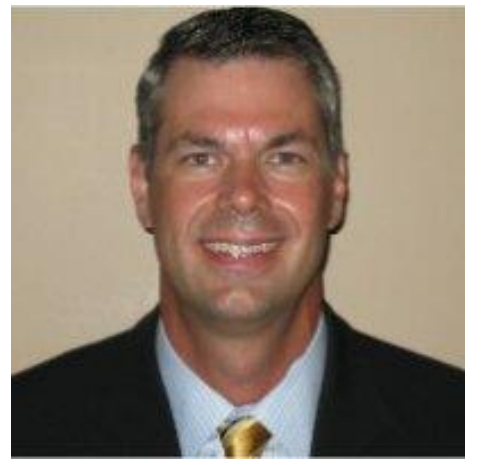

Mr. Jones is Director-Consulting Services at Schumacher Clinical Partners; Telehealth/Telemedicine Strategy \& Operations. He more than 15 years of experience leading clients through the challenging aspects of planning, forecasting, selecting, contracting, organizing, implementing and operationalizing technology to achieve meaningful change, benefits and opportunities.

Reference

1. Project Management Institute. The high cost of low performance. 2014. URL:

http://www.pmi.org/-/media/pmi/documents/public/pdf/learning/thoughtleadership/pulse/pulse-of-the-profession-2014.pdf

\section{Tags}

accountability, documented services, executive and physician involvement, informatics technologies, integration, integration of services, Jeffrey Jones, strategy, success criteria, successful telehealth program, Telehealth, telemedicine, telemedicine process improvement, telemedicine program implementation, what is telemedicine and how does it work 